\title{
The Effects of Increased Central Serotonergic Activity on Prepulse Inhibition and Habituation of the Human Startle Response
}

\author{
Kristian S Jensen', Bob Oranje ${ }^{*, 2}$, Malene Wienberg' and Birte Y Glenth $\varnothing \mathbf{j}^{\mathbf{1}}$ \\ 'Center for Neuropsychiatric Schizophrenia Research, Psychiatric Center Glostrup, University of Copenhagen, Glostrup, Denmark; ${ }^{2}$ Center for \\ Neuropsychiatric Schizophrenia Research, Psychiatry University Center Glostrup, Glostrup Hospital, Glostrup, Denmark
}

\begin{abstract}
Sensorimotor gating is critical to normal brain functioning, and disruptions are associated with certain mental illnesses, such as schizophrenia. Prepulse inhibition of the acoustic startle reflex (ASR) (PPI) is an operational measure of sensorimotor gating, of which evidence for a serotonergic modulation is currently inconsistent. In a double-blind placebo-controlled crossover design, I 8 healthy male volunteers received either placebo or a dose of $10 \mathrm{mg}$ of escitalopram (SSRI), after which they were tested in both PPI and habituation of the startle reflex paradigms. No significant differences between the two treatments were observed on PPI, although escitalopram was found to significantly delay habituation of the ASR. In the current study, escitalopram was found to delay habituation, but it did not affect PPI in healthy male volunteers. As escitalopram is a highly specific SSRI, the results suggest that an increased serotonergic activity disrupts habituation, but not PPI in healthy volunteers.
\end{abstract}

Neuropsychopharmacology (2007) 32, 2 I 17-2124; doi:I0.1038/sj.npp. I301350; published online I4 February 2007

Keywords: escitalopram; serotonin; habituation; PPI; sensorimotor gating; antidepressants

\section{INTRODUCTION}

The startle reflex is an abrupt response to a sudden and intense stimulus and can be elicited by several modalities of stimuli, for example sound and tactile stimuli. The reflex is a series of twitches of the orbicularis oculi muscles (eye blink component) and the large skeletal muscle groups. The reflex is not only restricted to humans, but also found in other vertebrates. In humans, the acoustic startle reflex (ASR) is usually assessed by electromyographic recordings of the eye blink component (Blumenthal et al, 2005). The ASR can be modulated through a variety of mechanisms, such as prepulse inhibition (PPI) and habituation.

PPI of the ASR is an attenuation of the startle amplitude by a non-startling prepulse preceding the startle-eliciting pulse by a certain amount of time, and is considered an operational measure of sensorimotor gating (eg Graham, 1975; Geyer et al, 1990; Swerdlow et al, 1994). Habituation is a reduction over time of the amplitude of the ASR, seen after repeated presentation of identical startle eliciting pulses, which is not a result of sensory adaptation or muscle

\footnotetext{
*Correspondence: Dr B Oranje, Center for Neuropsychiatric Schizophrenia Research, Psychiatry University Center Glostrup, Glostrup Hospital, Ndr. Ringvej, DK-2600 Glostrup, Denmark, Tel: + 45432 34638, Fax: + 45432 34653, E-mail: B.Oranje@cnsr.dk Received 25 July 2006; revised 44 December 2006; accepted 2 January 2007
}

fatigue, and is considered a form of non-associative learning (Christoffersen, 1997). Patients with schizophrenia have frequently been found to show disrupted PPI, when compared with healthy controls (eg Braff et al, 1978, 1992; Kumari et al, 2000; Parwani et al, 2000; Mackeprang et al, 2002).

The deficient gating is thought to lead to a flooding of cortical areas with sensory input, causing overpowering of processing capacities and thereby reducing the ability to focus on relevant stimuli. This is hypothesized to contribute to some of the core symptoms found in schizophrenic patients, such as deficits in information processing, attention, and thought disorder (Braff and Geyer, 1990; Perry et al, 1999). In longitudinal studies of patients with schizophrenia, reduced PPI has been shown to be consistently present over time, indicating it to be a stable trait of schizophrenia as opposed to a state phenomenon (Mackeprang et al, 2002; Ludewig et al, 2002). Similar gating deficits have been found with a higher prevalence in relatives of schizophrenic patients as well as individuals with schizotypal personality disorder, thereby suggesting that the deficits are vulnerability markers already present before outright symptoms of schizophrenia are observed (Braff, 1993; Cadenhead et al, 2000, 2002). In contrast to PPI, habituation shows less consistent results: some studies showing reduced habituation (eg Geyer and Braff, 1982; Parwani et al, 2000; Perry et al, 2002; Ludewig et al, 2003) and some failing to show habituation deficits in 
patients with schizophrenia (eg Braff et al, 1999; Kumari et al, 2000, 2002).

On the biochemical level, pharmacological modulation of PPI and habituation in healthy volunteers has been widely used as a model for gating deficits in schizophrenia, where different neurotransmitter systems, such as the dopaminergic (eg Abduljawad et al, 1998; Oranje et al, 2004) and glutamatergic (eg van Berckel et al, 1998; Oranje et al, 2002a; Abel et al, 2003) systems have been explored (for a review, see Braff et al, 2001). Furthermore, both typical and atypical antipsychotics have been tested in several clinical studies: Kumari et al (1999) reported normal PPI in schizophrenic patients treated with the atypical antipsychotic clozapine, when compared with healthy controls, whereas patients treated with typical antipsychotics showed reduced PPI. This has been replicated by Oranje et al (2002b); (for a review, see Kumari and Sharma, 2002). In contrast to clozapine, Duncan et al (2003a) found that patients treated with olanzapine had levels of PPI comparable to patients treated with haloperidol or patients who are not treated at all, whereas this group as a whole showed significantly less PPI compared with healthy controls. This indicates that not every atypical antipsychotic is capable of normalizing PPI deficits, which also seems to be confirmed by a similar study of this group (Duncan et al, 2003b). In addition, in a recent study by Kumari et al (2006), patients treated with olanzapine and risperidone showed levels of PPI that were not significantly different from either healthy controls or from patients treated with typical antipsychotics (who showed significantly less PPI than the healthy controls). Currently, however, there is only one double-blind, randomized longitudinal study in which an atypical antipsychotic (risperidone) was compared with a typical antipsychotic (zuclopenthixol) in drug naïve first-episode schizophrenia patients (Mackeprang et al, 2002). This study failed to show any difference with regards to PPI deficits. Clozapine has high affinity for the 5-HT receptor family, which may account for some of the superiority with regard to efficacy when compared to typical antipsychotics. More specific, antagonism of the 5$\mathrm{HT}_{2 \mathrm{~A}}$ receptor combined with weak $\mathrm{D}_{2}$ antagonism is a common profile of many atypical antipsychotic drugs, such as olanzapine, clozapine, and quetiapine (for a review, see Meltzer et al, 2003).

A considerable number of studies with rodents suggest a role for serotonergic modulation of PPI and habituation (Geyer et al, 2001). However, in a study by Vollenweider et al (1999), where human subjects and rats were tested under comparable settings and design, opposite effects of the 5-HT releaser, 3,4-methylenedioxymethamphetamine (MDMA or ecstasy) on PPI was obtained, thereby enhancing PPI in humans, but decreasing PPI in rats. This supports a species-specific effect of serotonergic modulation of the ASR, which has also been observed in studies with even closer related animal species such as rats and mice, where 8-OH-DPAT (5- $\mathrm{HT}_{1 \mathrm{a} / \mathrm{b}}$ agonist) increased PPI in mice (Dulawa et al, 1997) but decreased it in rats (Rigdon and Weatherspoon, 1992), thereby underlining the complexity to compare studies on the effects of serotonergic modulation of PPI across species. In humans, GouzoulisMayfrank et al (1998) found increased PPI following stimulation of 5-HT receptors by administration of psilocybin, a 5- $\mathrm{HT}_{1 / 2}$ agonist. Similarly, MDMA (among others a 5-HT releaser) appears to increase PPI in healthy humans (Vollenweider et al, 1999; Liechti et al, 2001). Other studies, however, failed to reproduce this enhancing effect of 5-HT on PPI (Phillips et al, 2000; Riba et al, 2002), probably owing to binding characteristics in general or different affinities for 5-HT receptors in specific.

In contrast to PPI, Quednow et al (2004) found decreased habituation after chronic administration of sertraline (SSRI) to patients with major depression. Results from our own laboratory (Hammer et al, 2007) have shown that imipramine, a noradrenergic/serotonergic reuptake inhibitor, reduces PPI but has no effect on habituation of the ASR, which suggests a detrimental effect of either serotonin or noradrenaline on PPI.

The current study was designed to further explore serotonergic modulation of PPI and habituation of the ASR by administering a highly selective serotonin reuptake inhibitor (escitalopram) to healthy, male volunteers, after which they were tested for PPI and habituation of the ASR. On the basis of our previous data (Hammer et al, 2007) - and the literature as mentioned above-our hypothesis was that increased serotonergic activity would neither influence PPI nor habituation. To monitor the effects of escitalopram, physiological (blood pressure), biochemical (serum escitalopram), and endocrine (serum cortisol) assessments were performed. As escitalopram is known to increase cortisol (Nadeem et al, 2004), an increase in the plasma level of this hormone was expected. Blood pressure was monitored, as orthostatic hypotension is one of the listed side effects of escitalopram.

\section{METHODOLOGY}

\section{Subjects}

The study was approved by the Committee for Biomedical Research Ethics, Copenhagen, with regard to the ethical principles for medical research involving human subjects as stated in the declaration of Helsinki (amendment of Washington, 2002). Written and oral information was given, after which written informed consent was obtained from all subjects. Male volunteers between 18 and 35 years of age, all non-smokers, were recruited via a university newspaper. Only physically healthy males who were not taking any prescription drugs and with a body mass index between 19 and 25, not showing alcohol and drug abuse nor personal or family history of psychiatric illness in firstdegree relatives were included. Physical and mental health history and absence of a history of drug and alcohol abuse were ascertained by use of the Schedule for Clinical Assessment in Neuropsychiatry, version 2.1 (SCAN) (Wing et al, 1990). Blood pressure was measured, and all subjects underwent a routine electrocardiogram recording. To screen for hearing deficits, subjects were tested at 500, 1000 , and $6000 \mathrm{~Hz}(40 \mathrm{~dB})$. Twenty-one subjects fulfilled the inclusion criteria, and were subsequently included in the study. Two subjects became severely nauseated during test day 1 , most likely owing to a vaso-vagal attack provoked by blood sampling, as the symptoms were reported during blood sampling. Both subjects were excluded, leaving 19 subjects who completed both test days. Owing to hardware 
failure, the PPI assessment of one treatment of a subject was lost, so the subject was entirely excluded from the analysis. Furthermore, one subject showed normal startle amplitudes during the habituation trials of the placebo treatment, but showed such rapid decline on subsequent trials of block 1 (strong habituation) that he was considered a nonresponder during PPI assessment (startle amplitudes to pulse alone trials below $20 \mu \mathrm{V}$ ). He was excluded from PPI, but not from habituation analysis. Two subjects showed an ASR to the loudest intensity prepulse $(85 \mathrm{~dB})$, as was observed on the $120 \mathrm{~ms}$ ISI prepulse-pulse trials. However, exclusion of these two from the analysis did not change the results; therefore, it was decided to include these two subjects in the analysis. The remaining 17 subjects in the PPI analysis had a mean age of 25.3 years $(S D=2.6$ years) and a mean body mass index of $23.2(\mathrm{SD}=1.2)$, whereas the remaining 18 subjects in the habituation analysis had a mean age of 25.2 years $(S D=2.6$ years $)$ and a mean body mass index of $23.2(\mathrm{SD}=1.2)$.

\section{Experimental Design}

In a balanced, double-blind, placebo-controlled cross-over design, healthy male volunteers were tested in a psychophysiological test battery (P50, PPI, selective attention, and mismatch negativity) on two occasions, separated by a minimum of 1 week: once after oral administration of placebo (calcium tablets) and once after oral administration of $10 \mathrm{mg}$ escitalopram (Cipralex $\left.{ }^{\circledR}\right)$. The volunteers had never before participated in psychophysiological research, and neither had ever received psychopharmacological medication before. The order of the treatments was balanced, that is, half of the subjects received placebo on the first test day and escitalopram on the second, whereas the other half received escitalopram on the first test day and placebo on the second test day. The order of the tests in both treatment sessions was such that subjects always started with the hearing test $(5 \mathrm{~min})$ followed by either the PPI ( $25 \mathrm{~min}$ ) or the P50 suppression $(21 \mathrm{~min}$ ) paradigm (the order of which was balanced over the subjects, yet for each subject the same for both treatment sessions), which was followed by the selective attention (13 min) paradigm, and finally the MMN (12 min) paradigm. Results of P50 suppression, selective attention, and mismatch negativity will be published elsewhere.

Subjects arrived at 0830 hours at the research ward at the Department of Psychiatry, Bispebjerg University Hospital, Copenhagen. Subjects were requested to fast from 2300 hours the previous day. On the first test day, subjects delivered a urine sample for toxicology screening (cannabis, cocaine, amphetamines). Following physiological assessments and blood sampling, $10 \mathrm{mg}$ of escitalopram or placebo was given orally. To ensure blindedness, both placebo and escitalopram tablets were put in identical opaque white gelatin capsules. The psychophysiological test battery was initiated at the expected time of $\mathrm{T}_{\text {Max }}$ for plasma level of escitalopram, that is $210 \mathrm{~min}$ after oral administration of the capsules (Gutierrez and Abramowitz, 2000). Serum cortisol and serum escitalopram were measured three times during each test day, whereas blood pressure was measured four times during each test day. In addition, two questionnaires were administered to assess for eventual adverse effects owing to treatment: the Adverse Symptom Checklist (ASC) and the Adverse Event Record (AER). Blood samples were drawn at baseline (before administration of the capsule), and at $210 \mathrm{~min}$ and $285 \mathrm{~min}$ after oral administration of medication (coinciding with the start and end of the psycho-physiological test battery). For cortisol sampling, $5 \mathrm{ml}$ of blood was taken in a gelatin-containing sample glass, and for escitalopram sampling, $10 \mathrm{ml}$ of blood was taken in a heparinized sample glass, which was kept at $5^{\circ} \mathrm{C}$ until the test day was complete after which they were centrifuged at 1500 r.p.m. for $10 \mathrm{~min}$ and stored at $-20^{\circ} \mathrm{C}$ until analysis.

\section{Assessment of PPI}

Subjects were seated in a comfortable armchair in a room with a sound level below $40 \mathrm{~dB}$ and situated adjacent to the control room. Subjects were instructed to sit still, to keep their eyes fixed on a spot on the wall directly in front of them and were asked to stay awake. PPI testing was initiated with $5 \mathrm{~min}$ of acclimatization to the background noise (70 $\mathrm{dB}$ white noise), after which three experimental blocks of stimuli were superimposed on the background noise. Blocks 1 and 3 were used to assess habituation of the ASR. The two blocks were identical and consisted of eight pulsealone trials (white noise with an intensity of $115 \mathrm{~dB}$ and a duration of $20 \mathrm{~ms}$, instant rise and fall) with randomized inter-trial intervals between 10 and $20 \mathrm{~s}$, to assess habituation. Block 2 consisted of 50 trials presented in a pseudorandomized order, to assess PPI. Prepulses consisted of bursts of white noise with intensities of either 6 or $15 \mathrm{~dB}$ above background with a duration of $20 \mathrm{~ms}$. Stimulus onset asynchrony (SOA) in prepulse-pulse trials was either 60 or $120 \mathrm{~ms}$, whereas inter-trial intervals were randomized between 10 and $20 \mathrm{~s}$. Randomized across the session, 10 pulse alone and 10 of each prepulse-pulse combination, based on SOA and intensity $(60 \mathrm{~ms} / 76 \mathrm{~dB}, 60 \mathrm{~ms} / 85 \mathrm{~dB}$, $120 \mathrm{~ms} / 76 \mathrm{~dB}, 120 \mathrm{~ms} / 85 \mathrm{~dB})$, were presented. All auditory stimuli were presented by a computer running Presentation ${ }^{\circledR}$ software (soundcard: Creative soundblaster ${ }^{\circledR}, 5.1$ ) and presented binaurally through stereo insert earphones (Eartone ABR). During the testing and experimental block, a continuous $70 \mathrm{~dB}$ white noise acted as a background noise. The software and hardware settings were calibrated by means of an artificial ear (Brüel and Kjær, type 2133) in order to make sure that the stimulus intensities at the subject's ears were the intended intensities. PPI assessment took approximately $25 \mathrm{~min}$, whereas the entire test battery took approximately $75 \mathrm{~min}$. Following off-line filtering of the data between 25 and $250 \mathrm{~Hz}$, startle amplitude was scored as the highest absolute amplitude in the time interval 20-100 ms after the startle eliciting pulse, whereas PPI was expressed as $((1-(\mathrm{PP} / \mathrm{PA})) \times 100 \%)$, with $\mathrm{PP}$ the average startle amplitude to prepulse-pulse trials and PA the average startle amplitude to pulse alone trials.

\section{Signal Recording}

Electroencephalography recordings were assessed with BioSemi ${ }^{\circledR}$ hardware using a cap with 64 Active Two electrodes (Metting van Rijn et al, 1996), arranged according to the 10-20 system. A total of eight facial 
electrodes were attached for electromyography recordings and reference purposes. Three reference electrodes were attached: two on the mastoids (one on the left and one on the right) and one on the tip of the nose. Of the remaining five electrodes, two electrodes were placed under the right eye, one aligned with the pupil, the other electrode positioned just laterally; for PPI and habituation assessment, two were placed at the outer canthus of each eye for horizontal electro-occulogram assessment, and one supraorbital (aligned with the pupil) for vertical electroocculogram assessment. Sampling started immediately before an experimental block started and lasted till the end of it. Auditory stimuli were presented binaurally through insert headphones. All signals were digitized online by computer at a rate of $4096 \mathrm{~Hz}$, and a low-pass setting of $1 / 5$ of the $\mathrm{AD}$ rate.

\section{Statistical Analysis}

All statistical analyses were performed with SPSS (for Windows, version 11.0). Effect of treatment on startle magnitude was analyzed using an analysis of variance (ANOVA) with factors 'treatment' (placebo or escitalopram) and 'stimtype' (pulse alone, prepulse-pulse $(60 \mathrm{~ms}, 76 \mathrm{~dB})$ prepulse-pulse $(120 \mathrm{~ms}, 76 \mathrm{~dB})$, prepulse-pulse $(60 \mathrm{~ms}$, $85 \mathrm{~dB})$, or prepulse-pulse $(120 \mathrm{~ms}, 85 \mathrm{~dB})$ ). PPI of the startle reflex was analyzed using ANOVA with factors 'treatment', 'prepulse intensity' (76 or $85 \mathrm{~dB}$ ), and 'SOA' (60 or $120 \mathrm{~ms}$ ). Furthermore, as studies of patients with schizophrenia have shown that the largest deficits are observed at $\mathrm{SOA}=120 \mathrm{~ms}$ and a prepulse intensity of $85 \mathrm{~dB}$ (Braff et al, 2001), a planned comparison of PPI at these settings between the two treatments was performed with a paired samples Student's $t$-test. Habituation was analyzed using ANOVA of mean startle amplitude in blocks 1 and 3 with factors 'treatment' and 'block' (block 1 or block 3). As two other studies from our laboratory, in which exactly the same habituation paradigm was used as in the current study, showed that the first four trials of the first habituation block displayed the most variation or decline in response amplitudes (similar to the current study, see Figure 3), a post hoc comparison between the startle amplitudes of trials 1 through 4 was performed with an ANOVA with factors 'treatment' and 'trial' (trial 1 through 4). In addition, a post hoc comparison was performed between the percentage magnitude of trial 2 relative to trial 1 (formula: $100 \times(\mathrm{am}-$ plitude trial 2/amplitude trial 1)) and the magnitude of trial 1 over both treatments, as previous research with a similar habituation paradigm had shown a sensitization in the response of healthy volunteers from trial 1 to 2 (Meincke et al, 2004). Analysis of blood pressure was carried out using ANOVA with factors 'treatment' and 'time' $(t=0 \mathrm{~h}$ or $t=210$ or $t=285 \mathrm{~min}$ ) of systolic and diastolic blood pressure separately. As escitalopram is known to increase cortisol levels (Nadeem et al, 2004), a planned comparison between cortisol levels in the two treatment groups at $t=210$ and $t=285 \mathrm{~min}$ was performed with a paired samples Student's $t$-test. Furthermore, to test whether serum levels of escitalopram were constant during psychophysiological testing, a paired samples Student's $t$-test was performed to compare the levels at $t=210 \mathrm{~min}$ and $t=285 \mathrm{~min}$.

\section{RESULTS}

\section{Biochemical Measurements}

There was no significant difference in plasma levels of escitalopram at times $210 \mathrm{~min}$ and $285 \mathrm{~min}$, indicating a steady state concentration (mean $=19.69 \mathrm{nmol} / \mathrm{l}$ at $t=210 \mathrm{~min}$, mean $=19.74 \mathrm{nmol} / 1$ at $t=285 \mathrm{~min}$ ) in the time interval between $t=210$ and $t=285 \mathrm{~min}$, which coincided with psychophysiological testing.

Similarly, baseline $(t=0)$ plasma levels of cortisol did not differ between the treatments. The planned comparison of cortisol levels at $t=210 \mathrm{~min}$ and $t=285 \mathrm{~min}$ revealed a significant increased level of serum cortisol in the escitalopram treatment at $t=210 \mathrm{~min}(t=2.84 ; p<0.05)$ (Figure 1). Analysis of systolic and diastolic blood pressure revealed neither an effect of treatment nor of time.

\section{PPI}

The ANOVA revealed a significant effect of stimtype $(\mathrm{F}(4,13)=9.73 ; p<0.001)$, indicating that prepulses indeed decreased startle amplitude, that is that PPI occurred. No effect of treatment was found on raw startle amplitude data. Furthermore, no significant effects of treatment, but main effects of 'SOA' and 'prepulse intensity', were found on percentage PPI, indicating an increased PPI with increasing SOA and prepulse intensity (Figure 2).

The planned comparison of percentage PPI in the prepulse-pulse trials with $\mathrm{SOA}=120 \mathrm{~ms}$ and prepulse intensity $=85 \mathrm{~dB}$ revealed no significant effect of treatment $(t=0.27 ; p=0.79)$.

\section{Habituation}

A significant effect of block was found $(F(1,17)=47.53$; $p<0.001$ ) indicating that subjects had higher startle amplitudes in block 1 than in block 3 . No effect of treatment was found. The post hoc comparison across trial 1 through 4 from block 1 showed a significant treatment $\times$ trial interaction effect $(\mathrm{F}(3,15)=3.72 ; p<0.035)$ with an effect size

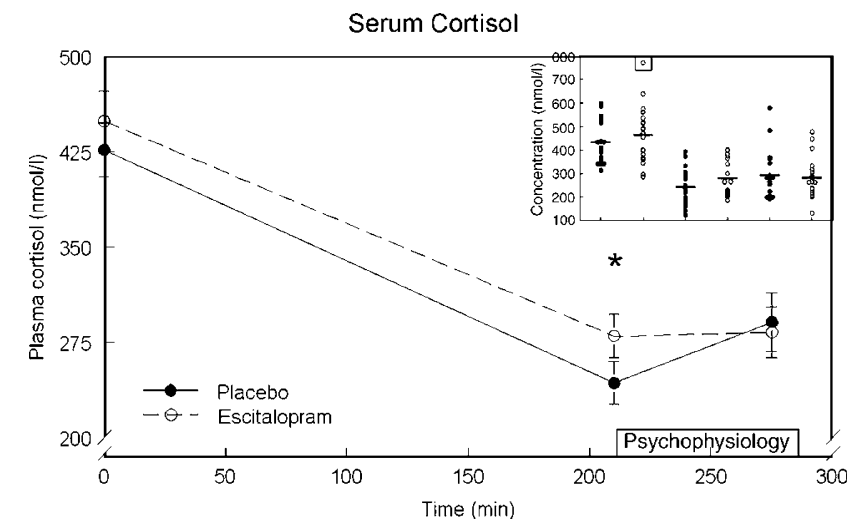

Figure I Serum cortisol levels ( \pm SEM). A significant increase in cortisol levels in the escitalopram treatment is observed at $t=210 \mathrm{~min}$. In the upper right corner, a box plot of the displayed data is shown, in which an obvious outlying (baseline) value was identified (marked in box). No change occurred in the statistical outcome on exclusion of this (baseline) value. The (baseline) value was excluded from the main graph. 


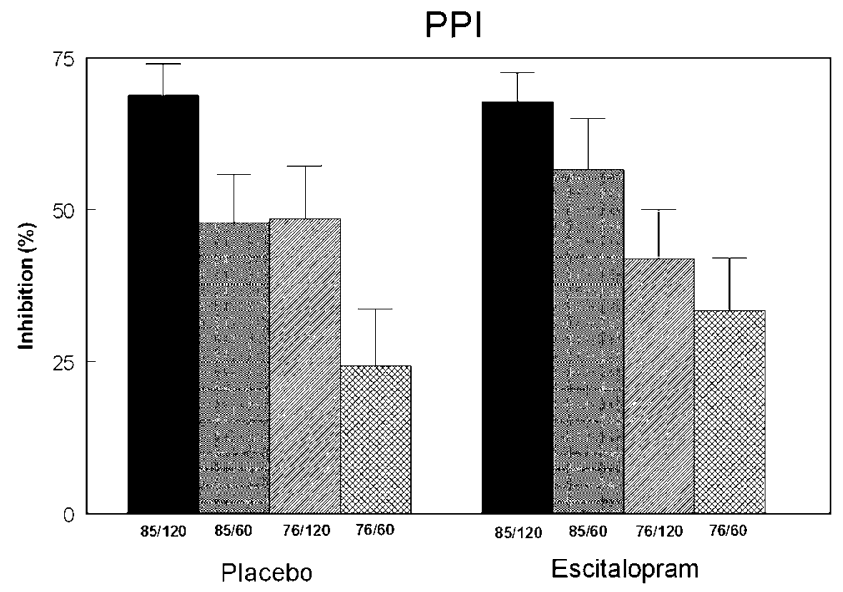

Figure 2 Main \% PPI ( \pm SEM) for all four different prepulse-pulse trials. A clear increase in \% PPI with increasing SOA and prepulse intensity is observed. No effect of treatment on \% PPI was found.

$\left(\eta_{\mathrm{p}}^{2}\right)$ of 0.43 , indicating a significant decrease in startle amplitude across trial 1 through 4 in the placebo treatment, but not in the escitalopram treatment (Figure 3). The post hoc analysis on percentage magnitude of trial 2 relative to trial 1 revealed no significant effects of sensitization $(\mathrm{F}(1,17)=3.12 ; \quad p=0.095), \quad$ treatment $\quad(\mathrm{F}(1,17)=0.80$; $p=0.38$ ), or a significant interaction effect.

\section{DISCUSSION}

The aim of this study was to examine the effects of increased central serotonergic activity on PPI and habituation of the ASR by administration of the SSRI escitalopram to a group of healthy male volunteers. The main findings of the study were that escitalopram had no effect on PPI, but reduced habituation of the first four presented trials.

Previous results from our laboratory (Hammer et al, 2007) suggested that an increase in either serotonergic or noradrenergic activity decreases sensorimotor gating, as acute administration of imipramine (a combined serotonergic and noradrenergic reuptake inhibitor) was found to decrease PPI in healthy volunteers. The result of the current study, in which no effect of escitalopram (highly specific SSRI) was found on the PPI of healthy male volunteers, suggests that increased serotonergic activity does not affect PPI, and accordingly that the detrimental effect of imipramine on PPI, as found in our previous study, was due to an increase in noradrenergic activity. The line of thought that increased serotonergic activity does not affect PPI would be consistent with the studies of Phillips et al (2000) and Liechti et al (2001) in which no effects of respectively fluvoxamine and citalopram (both SSRIs) were found on PPI in healthy subjects. The evidence that not only an acute increased serotonergic activity, but also a chronically increased serotonergic activity seems to have no effect on PPI, is supplied by the study of Quednow et al (2004), in which no effects of sertraline (SSRI) on PPI were found in a population of patients with major depression. The notion that the detrimental effects of imipramine on PPI in our previous study (Hammer et al, 2007) were due to

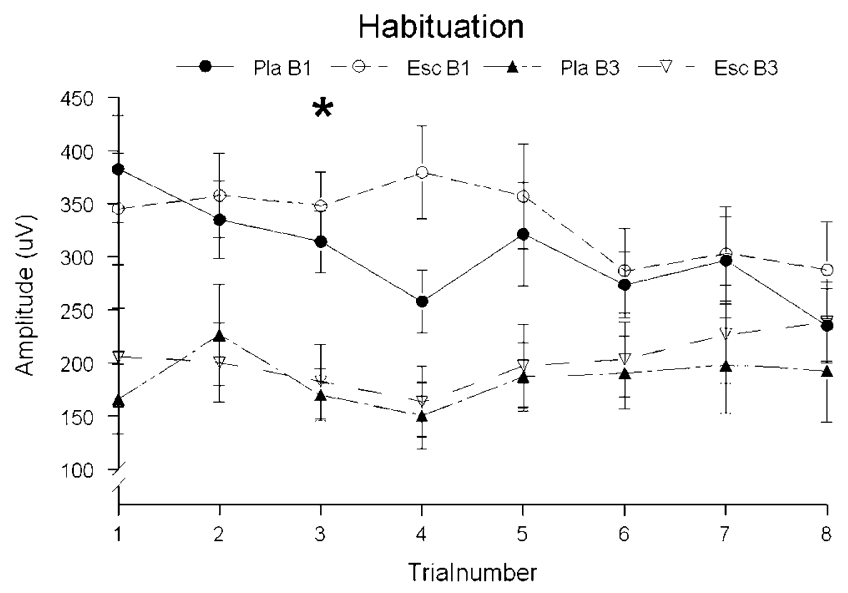

Figure 3 Mean startle amplitude $( \pm$ SEM) for the eight different trials of both the blocks I and 3. A significant effect of treatment was found on startle habituation over the first four trials of block I.

an increased noradrenergic activity would be consistent with the results from other studies in which noradrenaline was found to reduce human PPI (Oranje et al, 2004; Quednow et al, 2004), although a possible anticholinergic effect cannot be ruled out, as imipramine has affinity for this receptor too. The discrepancy between the current study and the studies of Liechti et al (2001) and Vollenweider et al (1999), in which MDMA (or 'ecstasy,' which is a serotonin releasing agent with similar effects on the dopaminergic and noradrenergic system) was found to increase PPI in healthy volunteers, might be attributable to the additional effects of MDMA on the dopaminergic and/or noradrenergic system.

The data from the current study, and two other studies from our lab in which exactly the same habituation paradigm was used as in the current study, show that the process of habituation of the ASR seems to take place mainly during very early testing, that is during the first four trials. Escitalopram was found to significantly delay this habituation process in the present study, indicating that habituation, in contrast with PPI, is affected by increased serotonergic activity (please note that this effect is moderate, as the effect size is only 0.43 ). In a longitudinal study, Quednow et al (2004) found that the SSRI sertraline reduced habituation in patients with major depression, which is consistent with the results of the current study. Liechti et al (2001) found ketanserin to increase habituation, which is well in line with our findings, as the compound is a $5 \mathrm{HTa} / \mathrm{c}$ antagonist, so an opposite effect of escitalopram is plausible. In contrast, Liechti et al (2001) found no effect of citalopram (SSRI) on habituation, probably owing to the fact that habituation was calculated as the decrement of mean startle amplitude from block 1 to 3 , whereby the habituation deficits observed in the current study (over the first four trials) would have been masked. Similarly, in animal studies, fluvoxetine (SSRI) reduced habituation in rats (Geyer and Tapson, 1988), whereas administration of the 5-HT1A/1B agonist RU24969 reduced habituation in mice (Dulawa and Geyer, 2000). In the current study, no sensitization effect was found, as opposed to the study of Meincke et al (2004) (NB: although the effect 
of sensitization appears to reach towards significance in the current study, it was only based on the responses of three subjects, of which one was a considerable outlier (an increase in the response to trial 2 of $486 \%$ compared with trial 1)). The discrepancy between the current study and the study of Meincke et al (2004) regarding sensitization might be explained by the difference in subject population: in the current study, only males were included, whereas in the study of Meincke et al (2004) both males and females were included. As there are numerous reports in literature describing a gender difference in both PPI (Swerdlow et al, 1993, 1999; Jovanovic et al, 2004; Kumari et al, 2004) and/or prepulse facilitation (Aasen et al, 2005), it is not inconceivable that gender differences might also exist in the processes of habituation and sensitization. Another explanation might be the difference in the intensity level of the background noise, which was slightly higher in the current study $(70 \mathrm{~dB})$ than in the study of Meincke et al (2004) $(65 \mathrm{~dB})$, which might have reduced the sensitization effect.

Antidepressant compounds are often added to antipsychotic treatment of patients with schizophrenia. A majority of antidepressants increase serotonergic or noradrenergic activity or both, so-called dual action compounds. Therefore, elucidating the effects of serotonergic and noradrenergic modulation of PPI has relevance to the treatment of patients with schizophrenia. The finding of the present study that serotonin has no effect on PPI might indicate that gating deficits in patients with schizophrenia will not worsen when they are treated with highly selective SSRIs such as escitalopram. The finding that escitalopram has a mild (first four trials only with a moderate effect size) negative effect on the process of habituation might indicate that already existing habituation deficits of patients that undergo treatment with escitalopram might intensify, which, in turn, might have a (minor) negative impact on the symptomatology of these patients.

There are certain limitations to the present study. Although a significantly higher serum cortisol level was observed after $210 \mathrm{~min}$ after administration of escitalopram and that this dose reduced habituation in the current study, whereas it increased processing and mismatch negativity (two other psychophysiological parameters of attention) (Oranje et al, submitted), the current dose of $10 \mathrm{mg}$ might have been too low to affect PPI. Another limitation might have been the level of specificity of escitalopram for the serotonergic system. However, as escitalopram is known to have a high specificity for the serotonergic system (Gutierrez and Abramowitz, 2000), it seems unlikely that this would have compromised the data. Furthermore, the data should be considered with the necessary caution, as participants received only one single dose of escitalopram. Whether the effect of escitalopram stays the same over time, like in the treatment of depressive or negative symptoms of schizophrenia, or will increase/alter over time, cannot be determined by the current study. Finally, it can be argued that the absence of effects of escitalopram on PPI is caused by the stimulation of serotonergic autoreceptors, resulting in a decreased serotonergic activity, rather than an increased activity, during the period of psychophysiological assessment. However, this is unlikely, as serum cortisol level was significantly increased at the start of the psychophysiological test battery. In addition, in a recent publication on the pharmacokinetics of escitalopram in rats, the level of 5HT in the hippocampus was still increased $210 \mathrm{~min}$ after administration of various doses of escitalopram (which corresponds with the start of the assessments in the current study) (Bundgaard et al, 2006).

In conclusion, in the present study, escitalopram did not affect PPI, which provides further evidence that a nonspecific increase in serotonergic activity does not affect human sensorimotor gating. Furthermore, escitalopram disrupted habituation of the startle reflex in the current study, which suggests that this process, in contrast to PPI, is sensitive to a nonspecific increase in central serotonergic activity. Future research should focus on the role of specific serotonergic receptor subtypes in PPI and habituation, and on the effects of a more chronic use of escitalopram on PPI and habituation of the startle reflex, such as for instance in the treatment of depressed and negative symptoms in schizophrenia.

\section{ACKNOWLEDGEMENTS}

This study was supported by an unrestricted grant from the Lundbeck foundation and by the Faculty of health Sciences from the University of Copenhagen. Furthermore, the study was approved by the Committee for Biomedical Research Ethics Copenhagen, and therefore complies with the current laws regarding experimental research of Denmark.

\section{REFERENCES}

Aasen I, Kolli L, Kumari V (2005). Sex effects in prepulse inhibition and facilitation of the acoustic startle response: implications for pharmacological and treatment studies. J Psychopharmacol 19: 39-45.

Abduljawad KA, Langley RW, Bradshaw CM, Szabadi E (1998). Effects of bromocriptine and haloperidol on prepulse inhibition of the acoustic startle response in man. J Psychopharmacol 12: 239-245.

Abel KM, Allin MP, Hemsley DR, Geyer MA (2003). Low dose ketamine increases prepulse inhibition in healthy men. Neuropharmacology 44: 729-737.

Blumenthal TD, Cuthbert BN, Filion DL, Hackley S, Lipp OV, van Boxtel A (2005). Committee report: guidelines for human startle eyeblink electromyographic studies. Psychophysiology 42: 1-15.

Braff DL (1993). Information processing and attention dysfunctions in schizophrenia. Schizophr Bull 19: 233-259.

Braff DL, Geyer MA, Swerdlow NR (2001). Human studies of prepulse inhibition of startle: normal subjects, patient groups, and pharmacological studies. Psychopharmacology (Berlin) 156: 234-258.

Braff DL, Grillon C, Geyer MA (1992). Gating and habituation of the startle reflex in schizophrenic patients. Arch Gen Psychiatry 49: 206-215.

Braff DL, Stone C, Callaway E, Geyer MA, Glick I, Bali L (1978). Prestimulus effects on human startle reflex in normals and schizophrenics. Psychophysiology 15: 339-343.

Braff DL, Swerdlow NR, Geyer MA (1999). Symptom correlates of prepulse inhibition deficits in male schizophrenic patients. Am J Psychiatry 156: 596-602.

Braff DL, Geyer MA (1990). Sensorimotor gating and schizophrenia: human and animal model studies. Arch Gen Psychiatry 47: 181-188.

Bundgaard C, Larsen F, Jørgensen M, Gabrielsson J (2006). Mechanistic model of acute autoinhibitory feedback action after 
administration of SSRIs in rats: application to escitalopraminduced effects on brain serotonin levels. Eur J Pharm Sci 29: 394-404.

Cadenhead KS (2002). Vulnerability markers in the schizophrenia spectrum: implications for phenomenology, genetics, and the identification of the schizophrenia prodrome. Psychiatr Clin North Am 25: 837-853.

Cadenhead KS, Swerdlow NR, Shafer KM, Diaz M, Braff DL (2000). Modulation of the startle response and startle laterality in relatives of schizophrenic patients and in subjects with schizotypal personality disorder: evidence of inhibitory deficits. Am J Psychiatry 157: 1660-1668.

Christoffersen GR (1997). Habituation: events in the history of its characterization and linkage to synaptic depression. A new proposed kinetic criterion for its identification. Prog Neurobiol 53: 45-66.

Dulawa SC, Geyer MA (2000). Effects of strain and serotonergic agents on prepulse inhibition and habituation in mice. Neuropharmacology 39: 2170-2179.

Dulawa SC, Hen R, Scearce Levie K, Geyer MA (1997). Serotonin1B receptor modulation of startle reactivity, habituation, and prepulse inhibition in wild-type and serotonin1B knockout mice. Psychopharmacology (Berlin) 132: 125-134.

Duncan E, Szilagyi S, Schwartz M, Kunzova A, Negi S, Efferen T et al (2003a). Prepulse inhibition of acoustic startle in subjects with schizophrenia treated with olanzapine or haloperidol. Psychiatry Res 120: 1-12.

Duncan EJ, Szilagyi S, Efferen TR, Schwartz MP, Parwani A, Chakravorty $S$ et al (2003b). Effect of treatment status on prepulse inhibition of acoustic startle in schizophrenia. Psychopharmacology (Berlin) 167: 63-71.

Geyer MA, Braff DL (1982). Habituation of the blink reflex in normals and schizophrenic patients. Psychophysiology 19: $1-6$.

Geyer MA, Krebs-Thomson K, Braff DL, Swerdlow NR (2001). Pharmacological studies of prepulse inhibition models of sensorimotor gating deficits in schizophrenia: a decade in review. Psychopharmacology (Berlin) 156: 117-154.

Geyer MA, Swerdlow NR, Mansbach RS, Braff DL (1990). Startle response models of sensorimotor gating and habituation deficits in schizophrenia. Brain Res Bull 25: 485-498.

Geyer MA, Tapson GS (1988). Habituation of tactile startle is altered by drugs acting on serotonin-2 receptors. Neuropsychopharmacology 1: 135-147.

Gouzoulis-Mayfrank E, Heekeren K, Thelen B, Lindenblatt H, Kovar KA, Sass H et al (1998). Effects of the hallucinogen psilocybin on habituation and prepulse inhibition of the startle reflex in humans. Behav Pharmacol 9: 561-566.

Graham FK (1975). The more or less startling effects of weak prestimulation. Psychophysiology 12: 238-248.

Gutierrez MM, Abramowitz W (2000). Pharmacokinetic comparison of oral solution and tablet formulations of citalopram: a single-dose, randomized, crossover study. Clin Ther 22: $1525-1532$.

Hammer TB, Oranje B, Glenthoj B (2007). The effects of imipramine on P50 suppression, prepulse inhibition and habituation of the startle response in humans. Int J Neuropsychopharmacol 25: 1-9.

Jovanovic T, Szilagyi S, Chakravorty S, Fiallos AM, Lewison BJ, Parwani A et al (2004). Menstrual cycle phase effects on prepulse inhibition of acoustic startle. Psychophysiology 41: 401-406.

Kumari V, Aasen I, Sharma T (2004). Sex differences in prepulse inhibition deficits in chronic schizophrenia. Schizophr Res 69: 219-235.

Kumari V, Antonova E, Geyer MA, ffytche D, Williams SC, Sharma T (2006). A fMRI investigation of startle gating deficits in schizophrenia patients treated with typical or atypical antipsychotics. Int J Neuropsychopharmacol 1-15.
Kumari V, Sharma T (2002). Effects of typical and atypical antipsychotics on prepulse inhibition in schizophrenia: a critical evaluation of current evidence and directions for future research. Psychopharmacology (Berlin) 162: 97-101.

Kumari V, Soni W, Mathew VM, Sharma T (2000). Prepulse inhibition of the startle response in men with schizophrenia- effects of age of onset of illness, symptoms, and medication. Arch Gen Psychiatry 57: 609-614.

Kumari V, Soni W, Sharma T (1999). Normalization of information processing deficits in schizophrenia with clozapine. Am J Psychiatry 156: 1046-1051.

Kumari V, Soni W, Sharma T (2002). Prepulse inhibition of the startle response in risperidone-treated patients: comparison with typical antipsychotics. Schizophr Res 55: 139-146.

Liechti ME, Geyer MA, Hell D, Vollenweider FX (2001). Effects of MDMA (ecstasy) on prepulse inhibition and habituation of startle in humans after pretreatment with citalopram, haloperidol, or ketanserin. Neuropsychopharmacology 24: 240-252.

Ludewig K, Geyer MA, Etzensberger M, Vollenweider FX (2002). Stability of the acoustic startle reflex, prepulse inhibition, and habituation in schizophrenia. Schizophr Res 55: 129-137.

Ludewig K, Geyer MA, Vollenweider FX (2003). Deficits in prepulse inhibition and habituation in never-medicated, firstepisode schizophrenia. Biol Psychiatry 54: 121-128.

Mackeprang T, Kristiansen KT, Glenthoj BY (2002). Effects of antipsychotics on prepulse inhibition of the startle response in drug-naive schizophrenic patients. Biol Psychiatry 52: 863-873.

Meincke U, Light GA, Geyer MA, Braff DL, Gouzoulis-Mayfrank E (2004). Sensitization and habituation of the acoustic startle reflex in patients with schizophrenia. Psychiatry Res 126: 51-61.

Meltzer HY, Li Z, Kaneda Y, Ichikawa J (2003). Serotonin receptors: their key role in drugs to treat schizophrenia. Prog Neuropsychopharmacol Biol Psychiatry 27: 1159-1172.

Metting van Rijn AC, Kuiper AP, Dankers TE, Grimbergen CA (1996). Low-cost active electrode improves the resolution in biopotential recordings. Proceedings of the 18th Annual International Conference of the IEEE Engineering in Medicine and Biology Society, Amsterdam, The Netherlands, Track 12 3-3.

Nadeem HS, Attenburrow MJ, Cowen PJ (2004). Comparison of the effects of citalopram and escitalopram on 5-Ht-mediated neuroendocrine responses. Neuropsychopharmacology 29: 1699-1703.

Oranje B, Gispen-de Wied CC, Verbaten MN, Kahn RS (2002a). Modulating sensory gating in healthy volunteers. The effects of ketamine and haloperidol. Biol Psychiatry 52: 887-895.

Oranje B, Jensen KS, Wienberg M, Glenthoj BY. The effects of increased serotonergic activity on psychophysiological parameters of human selective attention (submitted).

Oranje B, Oel CJ, Gispen-de Wied CC, Verbaten MN, Kahn RS (2002b). Effects of typical and atypical antipsychotics on the prepulse inhibition of the startle reflex in patients with schizophrenia. J Clin Psychopharmacol 4: 359-365.

Oranje B, Verbaten MN, Kemner C, Kahn RS (2004). Modulating sensorimotor gating in healthy volunteers: the effects of desipramine and haloperidol. Psychiatry Res 127: 195-205.

Parwani A, Duncan EJ, Bartlett E, Madonick SH, Efferen TR, Rajan $\mathrm{R}$ et al (2000). Impaired prepulse inhibition of acoustic startle in schizophrenia. Biol Psychiatry 47: 662-669.

Perry W, Feifel D, Minassian A, Bhattacharjie I, Braff DL (2002). Information processing deficits in acutely psychotic schizophrenia patients medicated and unmedicated at the time of admission. Am J Psychiatry 159: 1375-1381.

Perry W, Geyer MA, Braff DL (1999). Sensorimotor gating and thought disturbance measured in close temporal proximity in schizophrenic patients. Arch Gen Psychiatry 56: 277-281.

Phillips MA, Langley RW, Bradshaw CM, Szabadi E (2000). The effects of some antidepressant drugs on prepulse inhibition of 
the acoustic startle (eyeblink) response and the N1/P2 auditory evoked response in man. J Psychopharmacol 14: 40-45.

Quednow BB, Kuhn KU, Stelzenmueller R, Hoenig K, Maier W, Wagner M (2004). Effects of serotonergic and noradrenergic antidepressants on auditory startle response in patients with major depression. Psychopharmacology (Berlin) 175: 399-406.

Riba J, Rodriguez-Fornells A, Barbanoj MJ (2002). Effects of ayahuasca on sensory and sensorimotor gating in humans as measured by P50 suppression and prepulse inhibition of the startle reflex, respectively. Psychopharmacology (Berlin) 165: $18-28$.

Rigdon GC, Weatherspoon JK (1992). 5-Hydroxytryptamine 1a receptor agonists block prepulse inhibition of acoustic startle reflex. J Pharmacol Exp Ther 263: 486-493.

Swerdlow NR, Auerbach P, Monroe SM, Hartston H, Geyer MA, Braff DL (1993). Men are more inhibited than women by weak prepulses. Biol Psychiatry 34: 253-260.
Swerdlow NR, Braff DL, Taaid N, Geyer MA (1994). Assessing the validity of an animal model of deficient sensorimotor gating in schizophrenic patients. Arch Gen Psychiatry 51: 139-154.

Swerdlow NR, Geyer MA, Hartman PL, Sprock J, Auerbach PP, Cadenhead K et al (1999). Sex differences in sensorimotor gating of the human startle reflex: all smoke? Psychopharmacology (Berlin) 146: 228-232.

van Berckel BN, Oranje B, van Ree JM, Verbaten MN, Kahn RS (1998). The effects of low dose ketamine on sensory gating, neuroendocrine secretion and behavior in healthy human subjects. Psychopharmacology (Berlin) 137: 271-281.

Vollenweider FX, Remensberger S, Hell D, Geyer MA (1999). Opposite effects of 3,4-methylenedioxymethamphetamine (MDMA) on sensorimotor gating in rats versus healthy humans. Psychopharmacology (Berlin) 143: 365-372.

Wing JK, Babor T, Brugha T, Burke J, Cooper JE, Giel R et al (1990). SCAN. Schedules for Clinical Assessment in Neuropsychiatry. Arch Gen Psychiatry 47: 589-593. 